\title{
Biomimicry for injectable mesh nanoelectronics
}

\author{
Clementene Clayton ${ }^{1} \&$ Bozhi Tian*,1 \\ ${ }^{1}$ Department of Chemistry, the James Franck Institute, the University of Chicago, Chicago, IL 60637, USA \\ *Author for correspondence: btian@uchicago.edu
}

First draft submitted: 19 June 2019; Accepted for publication: 2 August 2019; Published online: 14 August 2019

\section{Background}

Throughout numerous biological systems lie mesh-like structures, such as extracellular matrices and complex nervous networks, that modulate the biophysical and biochemical processes behind them [1]. For example, the astrocyte network in the CNS plays an important role in controlling electrophysiological activities in neurons; when these signals go awry, severe biological implications arise in the form of debilitating neurological disorders. To better understand how these networks function, modern technology has worked to accurately measure and understand these signals by probing and imaging. However, many of these methods are limited in their abilities due to issues such as device bulkiness, depth/volume of access, immune responses and durability [2].

Many recent scientific advancements can be credited to biomimicry or bioinspired technologies [3,4]. Consequently, biointerface designs have improved in exciting new ways $[1,3]$. For example, artificial nanostructures have been developed as a means of targeting and recording information from the nanoscale interactions within biological systems while mimicking the functional processes of biological nanowires [1]. Semiconductors, metals and conducting polymers are usually the materials of choice [5-9] because of their ability to perform functions of sensing and modulation, rather than simply exhibiting biochemical affinities or structural support.

\section{Biomedical applications of mesh electronics}

Researchers have used several ideas of biomimicry and developed an injectable mesh nanoelectronic system [10-16], showing neural signal monitoring down to the single-neuron level in the brains of mice. The use of this mesh technology could allow for high spatiotemporal resolution of aging studies that have not been possible before [11,14]. The minimal immune response and highly integrated nature of the mesh electronics system could open the horizons for a better understanding of and new treatments for debilitating neurodegenerative diseases $[11,13,14,16]$. Current techniques for targeting diseases like Parkinson's either lack specificity or severely disturb brain tissue in a manner that prevents seamless treatment. The needle-based injection coupled with the micro- or nanoscale features of these mesh electronics allow for the interfacing and recording on a single-neuron scale with little to no chronic immune response, thereby giving an accurate depiction of activity within the brain.

\section{Development of mesh nanoelectronics}

The technology for this mesh bioelectronics system was first developed in 2012 [17]. The conception of a flexible macroporous nanoelectronic scaffold was achieved as a potential means for mimicry of the 3D macroporous structures of extracellular matrices in tissues [17-19]. These scaffolds have been used for culture and concomitant electrical recordings of engineered neural, cardiac and vascular tissues. The biocompatibility of the scaffold can be improved through surface functionalization or hybridization of the mesh nanoelectronics with existing polymeric biomaterials into an integrated scaffold.

The ability for injection of the mesh structure through syringe injection was then illustrated by Liu et al. in 2015 [10]. Previously, there existed very limited approaches for minimally invasive delivery of 3D structures into neural networks within the brain [20]. Liu et al. showed how mesh structures, with 2-3 orders of magnitude the size of the injection needle diameter, could be packed and inserted into both hydrogels and live rodent brains [10]. The results of these trials demonstrated reliable monitoring with minimal immune response in the brain tissue $[10,13,14]$. 
This example represents a breakthrough in mesh electronics as it solved two challenges at that time: minimally invasive device delivery into the brain and electrical recording in vivo.

In the same year, Xie et al. developed a means of delivering a mesh probe by freezing the material to retain a rigid structure for penetration [15]. The mesh would eventually become soft after implantation due to the change in temperature. This, too, illustrated the ability to record single-unit action potentials within rodent brains, as well as the unique feature of neural tissue filling in the pores of the mesh for a highly integrated system.

From 2016 to 2018, studies fine-tuned the injectable mesh electronics to demonstrate both long-term and single unit recording [11-14,21]. Past methods of neural recording with typical brain probes have been limited to an observation period of days or weeks. The research done by Fu et al. proved the ability of the mesh nanoelectronics to monitor in vivo a single neuron or neural circuit in stable chronic manner over a period of at least 8 months [14], paving the way for neural aging and developmental studies. Another recent study by Hong et al. detailed how the mesh was injected into the retina of awake live mice to study neural circuitry over an at least 2-week period without compromising normal eye function [12]. Total 16-channel recordings from different types of single retinal ganglion cells were documented according to visual stimuli and circadian rhythm.

This year, Yang et al. evolved the injectable mesh to act as a biomimicry of neurons [16]. These neuron-like electronics (NeuE) are nearly indistinguishable from neurons in 3D imaging. The NeuE themselves are composed of building blocks that mimic the structure and mechanical properties of a neuron, thereby allowing for a seamless biointerface with minimal immune response and maintained stable recording of single neurons over extended periods of time. Not only do NeuE offer a pathway for neural electronic interface, but they have been shown to stimulate migration of new born neural cells, creating the potential for new regenerative medicine.

Other researchers have also played important roles in this field. In 2017, Luan et al. published a paper on a stable and chronic neural probe called a nanoelectronic thread [22]. This technique showed minimal glial scars or neural degradation with long-term single neuron recording. In 2013, Kim et al. contributed to the field through their pioneering work on injectable device-enabled optogenetics [20]. They demonstrated that injectable cellular scale light emitting diodes can elicit brain activities and behavior control in vivo [20]. These devices are biocompatible while also maintaining wireless capability, allowing for free movement of a live animal. Other significant flexible neural bioelectronics [9,23-27] include NeuroGrid [9] system that recorded both local field potentials and action potentials from the surface of brain, high density and flexible silicon nanomembrane transistor arrays that resolved seizure patterns [25], and graphene-based multiplexed and multifunctional platforms [26,27].

\section{Current challenges}

While injectable mesh electronics have surpassed many other forms of neural recording units in their biocompatibility and spatiotemporal resolution, there are still limitations to their capabilities. The needle used to insert the mesh into brain tissue is large enough to acutely perturb surrounding cytoarchitecture directly after injection. This could be mitigated through the use of needles with smaller diameters and optimization of mesh designs. Additionally, the mesh electronics are often limited in only being able to monitor relatively small numbers of electrodes over long periods of time. The electronics themselves can also be extended beyond silicon or metals. The use of open-mesh structures has pushed the technology closer to seamless biointegration, but its purely foreign composition may limit the extent of biocompatibility.

\section{Future perspective}

The drive for this mesh nanoelectronics technology lies in the desire to better understand and modulate biological, and especially neural activities. There are still a number of potential directions for growth. For example, in order to fully understand the brain and its underlying functions, numerous individual neurons must be monitored or modulated simultaneously, something this technology may allow scientists to accomplish in a minimally invasive way. These measurements also need to be conducted over different temporal scales to capture the dynamic behavior of the brain, whether that is in regard to millisecond signal transmissions or long-term development. The active components of the current devices are inorganic in their composition and could be hybridized with organic or biological materials to generate new standards of seamless integration and biological control [4,7,28-30]. The electronics' surface could be engineered to mimic the structural and functional properties of glial cells and their network, fashioning a novel form of neural interaction along a tight biointerface. The device modalities can be expanded to include complex electrical stimulation, drug delivery, imaging, tissue sample extraction, and ultimately a closed-loop system. These developments would enable future precision electronic medicine for complex and 
relatively poorly understood neurodegenerative diseases, as well as a better understanding of processes of memory and fundamental brain functions [4].

\section{Financial \& competing interests disclosure}

The authors have no relevant affiliations or financial involvement with any organization or entity with a financial interest in or financial conflict with the subject matter or materials discussed in the manuscript. This includes employment, consultancies, honoraria, stock ownership or options, expert testimony, grants or patents received or pending, or royalties.

No writing assistance was utilized in the production of this manuscript.

\section{References}

1. Tian BZ, Lieber CM. Nanowired bioelectric interfaces. Chem. Rev. 119(15), 9136-9152 (2019).

2. Hong GS, Lieber CM. Novel electrode technologies for neural recordings. Nat. Rev. Neurosci. 20, 330-345 (2019).

3. Luo ZQ, Weiss DE, Liu QY, Tian BZ. Biomimetic approaches toward smart bio-hybrid systems. Nano Res. 11(6), 3009-3030 (2018).

4. Acaron Ledesma H, Li XJ, Carvalho-De-Souza J, Wei W, Bezanilla F, Tian BZ. An atlas of nano-enabled neural interfaces. Nat. Nanotechnol. 14, 645-657 (2019).

5. Someya T, Bao ZN, Malliaras GG. The rise of plastic bioelectronics. Nature 540(7633), 379-385 (2016).

6. Wang SH, Oh JY, Xu J, Tran H, Bao Z. Skin-inspired electronics: an emerging paradigm. Acc. Chem. Res. 51(5), 1033-1045 (2018).

7. Jiang YW, Tian BZ. Inorganic semiconductor biointerfaces. Nat. Rev. Mater. 3(12), 473-490 (2018).

8. Ferro MD, Melosh NA. Electronic and ionic materials for neurointerfaces. Adv. Funct. Mater. 28(12), 1704335 (2018).

9. Khodagholy D, Gelinas JN, Thesen T et al. NeuroGrid: recording action potentials from the surface of the brain. Nat. Neurosci. 18(2), 310-315 (2015).

10. Liu J, Fu TM, Cheng Z et al. Syringe-injectable electronics. Nat. Nanotechnol. 10(7), 629-636 (2015).

11. Fu TM, Hong GS, Viveros RD, Zhou T, Lieber CM. Highly scalable multichannel mesh electronics for stable chronic brain electrophysiology. Proc. Natl Acad. Sci. USA 114(47), E10046-E10055 (2017).

12. Hong GS, Fu TM, Qiao M et al. A method for single-neuron chronic recording from the retina in awake mice. Science 360(6396), 1447-1451 (2018).

13. Zhou T, Hong GS, Fu TM et al. Syringe-injectable mesh electronics integrate seamlessly with minimal chronic immune response in the brain. Proc. Natl Acad. Sci. USA 114(23), 5894-5899 (2017).

14. Fu TM, Hong GS, Zhou T, Schuhmann TG, Viveros RD, Lieber CM. Stable long-term chronic brain mapping at the single-neuron level. Nat. Meth. 13(10), 875-882 (2016).

15. Xie C, Liu J, Fu TM, Dai XC, Zhou W, Lieber CM. Three-dimensional macroporous nanoelectronic networks as minimally invasive brain probes. Nat. Mater. 14(12), 1286-1292 (2015).

16. Yang X, Zhou T, Zwang TJ et al. Bioinspired neuron-like electronics. Nat. Mater. 18(5), 510-517 (2019).

17. Tian BZ, Liu J, Dvir T et al. Macroporous nanowire nanoelectronic scaffolds for synthetic tissues. Nat. Mater. 11(11), 986-994 (2012).

18. Tian BZ, Cohen-Karni T, Qing Q, Duan XJ, Xie P, Lieber CM. Three-dimensional, flexible nanoscale field-effect transistors as localized bioprobes. Science 329(5993), 830-834 (2010).

19. Dai XC, Hong GS, Gao T, Lieber CM. Mesh nanoelectronics: seamless integration of electronics with tissues. Acc. Chem. Res. 51(2), 309-318 (2018).

20. Kim TI, Mccall JG, Jung YH et al. Injectable, cellular-scale optoelectronics with applications for wireless optogenetics. Science 340(6129), 211-216 (2013).

21. Hong GS, Yang X, Zhou T, Lieber CM. Mesh electronics: a new paradigm for tissue-like brain probes. Curr. Opin. Neurobiol. 50, 33-41 (2018).

22. Luan L, Wei XL, Zhao ZT et al. Ultraflexible nanoelectronic probes form reliable, glial scar-free neural integration. Sci. Adv. 3(2), e1601966 (2017).

23. Khodagholy D, Doublet T, Quilichini P et al. In vivo recordings of brain activity using organic transistors. Nat. Commun. 4, 1575 (2013).

24. Kim D-H, Viventi J, Amsden JJ et al. Dissolvable films of silk fibroin for ultrathin conformal bio-integrated electronics. Nat. Mater. 9(6), 511-517 (2010).

25. Viventi J, Kim D-H, Vigeland L et al. Flexible, foldable, actively multiplexed, high-density electrode array for mapping brain activity in vivo. Nat. Neurosci. 14(12), 1599-1605 (2011).

26. Yin $\mathrm{RK}, \mathrm{Xu} \mathrm{Z}, \mathrm{Mei} \mathrm{M}$ et al. Soft transparent graphene contact lens electrodes for conformal full-cornea recording of electroretinogram. Nat. Commun. 9, 2334 (2018). 


\section{Editorial Clayton \& Tian}

27. Kuzum D, Takano H, Shim E et al. Transparent and flexible low noise graphene electrodes for simultaneous electrophysiology and neuroimaging. Nat. Commun. 5, 5259 (2014).

28. Jiang YW, Carvalho-De-Souza JL, Wong RC et al. Heterogeneous silicon mesostructures for lipid-supported bioelectric interfaces. Nat. Mater. 15(9), 1023-1030 (2016).

29. Jiang YW, Li XJ, Liu B et al. Rational design of silicon structures for optically controlled multiscale biointerfaces. Nat. Biomed. Eng. 2(7), 508-521 (2018).

30. Parameswaran R, Carvalho-De-Souza JL, Jiang YW et al. Photoelectrochemical modulation of neuronal activity with free-standing coaxial silicon nanowires. Nat. Nanotechnol. 13(3), 260-266 (2018). 